\title{
Proliferación celular en la mucosa de la vesícula biliar no tumoral en colecistitis crónicas litiásicas. Expresión de Ki-67 en matrices de tejidos
}

\author{
Iván Roa $E^{1}$, Xabier Elorza $D^{2}$, Soledad Lantadilla $H^{1}$, \\ Gilda Ibacache $\mathbf{S}^{1}$, Xabier de Aretxabala $\mathbf{U}^{3}$. \\ Immunohistochemical expression of \\ Ki-67 as a marker of proliferation in \\ gallbladder mucosa samples with or \\ without cancer
}

Background: There is paucity of knowledge on the proliferative features of normal or chronically inflamed gallbladder and the mechanisms of development of gallbladder cancer. Aim: To study the proliferation features of non tumoral gallbladder mucosa through the expression of Ki-67 antigen in tissue micro array analysis. Material and methods: The immunohistochemical expression of Ki-67 in tissue micro array was studied in 96 samples of non tumoral gallbladder mucosa (coming from 74 females aged $45 \pm 16$ years and 22 males aged $53 \pm 16$ years) and 102 samples of gallbladder cancer (coming from 84 females aged $62 \pm 14$ years and 18 males aged $70 \pm 13$ years). Results: The staining index of Ki-67 expression was $19 \pm 25 \%$ (range $0 \%-89 \%$ ) in samples of non tumoral mucosa and $46 \pm 29 \%$ (range $3 \%-98 \%$ ) in gallbladder cancer ( $\mathrm{p}<0.01$ ). Ki-67 was expressed in less than $10 \%$ of epitelial cells in $55 \%$ of non tumoral mucosa samples and $6 \%$ of gallbladder cancer samples. Seventy five percent of gallbladder cancer samples had a staining index of more than $20 \%$. An expression of Ki-67 over $20 \%$ or $50 \%$ was observed in $25 \%$ and $15 \%$ of non tumoral mucosa samples, respectively. Conclusions: Non tumoral gallbladder mucosa samples have a high proliferation index, measured using Ki-67 immunohistochemical expression. There is a group of samples with cellular hyper-proliferation that may be related to the pathogenesis of gallbladder cancer (Rev Méd Chile 2009; 137: 881-7).

(Key words: Gallbladder neoplasms; Ki-67 antigen; Microarray analysis)

Recibido el 6 de enero, 2009. Aceptado el 28 de mayo, 2009.

Trabajo financiado por Proyectos Fondecyt 1060375 y Bicentenario PBCT6

${ }^{1}$ Servicio de Anatomía Patológica, Clínica Alemana de Santiago. Facultad de Medicina Universidad del Desarrollo. Santiago de Chile. ${ }^{2}$ Servicio de Anatomía Patológica, Hospital San Camilo, San Felipe. ${ }^{3}$ Departamento de Cirugía, Clínica Alemana de Santiago.

Correspondencia a: Dr. Iván Roa E. Avenida Vitacura 5951,

Vitacura. Santiago de Chile. E mail: iroa@alemana.cl

Abreviaciones utilizadas:

MNT =mucosa vesicular no tumoral. CVB =cáncer de la vesícula biliar. TMA =matrices de tejidos. DE =desviación estándar 
$E^{1}$ cáncer de la vesícula biliar (CVB) continúa siendo uno de los grandes problemas en la patología tumoral maligna de la población chilena ${ }^{1,2}$. A nivel mundial nuestro país muestra una de las más altas tasas de incidencia y mortalidad ${ }^{3-}$ 5. A pesar de ello, son escasos los estudios sobre esta neoplasia, por lo cual, el grado de comprensión acerca de su patogenia es menor que en otras neoplasias ${ }^{6}$. La baja frecuencia con que este tumor se observa en países desarrollados, es sin duda la explicación a esta situación ${ }^{7-9}$.

Respecto de la histogénesis del CVB son múltiples los aspectos desconocidos, especialmente aquellos relacionados con las lesiones precurso$\operatorname{ras}^{10}$. La evidencia morfológica y molecular sugiere que la secuencia displasia-carcinoma sería la vía más importante en el desarrollo del CVB ${ }^{10,11}$.

El proceso por el cual se produciría la transformación desde la mucosa normal hacia la displasia, probablemente a través de la metaplasia de tipo gástrica o intestinal, ha recibido poca atención. Aun menos son los estudios relacionados con el proceso de proliferación celular del epitelio de la mucosa vesicular, que es en último término el sustrato de las lesiones preneoplásicas y del CVB ${ }^{12-14}$. Las caracteństicas proliferativas de la mucosa vesicular no tumoral (MNT) crónicamente inflamada, así como su relación con lesiones premalignas del epitelio de la mucosa vesicular aún no son conocidas ${ }^{13,15-18}$.

Distintos marcadores han sido utilizados como índices de proliferación celular, entre los cuales se destacan la timidina tritiada y la bromodeoxiuridina. Ambas se incorporan al ADN en la fase de síntesis y posteriormente son medidas a través de autorradiografía, citometría de flujo o inmunohistoquímica ${ }^{19,20}$. También se utilizan antígenos que se expresan durante algunas fases del ciclo celular como el PCNA ("Proliferating Cellular Nuclear Antigen") o el Ki67. La ventaja de estos últimos antígenos es que pueden ser medidos in situ en tejidos fijados en formalina e incluidos en parafina. En la actualidad el antígeno Ki67 se emplea rutinariamente en la evaluación del grado de proliferación celular como factor pronóstico en algunos tumores malignos ${ }^{21,22}$.

El antígeno Ki67 es una proteína nuclear codificada por un gen en el cromosoma 10, definida por su reactividad con el anticuerpo monoclonal Ki-67. Dos isoformas de 345 y $395 \mathrm{kDa}$ han sido identificadas. Ki67 es expresado durante todas las fases activas del ciclo celular (fase G1 tardío, S, G2 y mitosis) y está ausente en la Fase G0. Durante la interfase Ki67 es detectado dentro del núcleo, en cambio, en la mitosis la mayonía de estas proteínas son re-localizadas en la superficie de los cromosomas. Ki67 es rápidamente degradado cuando la célula entra a un estado noproliferativo o durante la reparación del ADN.

El objetivo de este trabajo es estudiar algunas caracteństicas de proliferación de la MNT, a través de la expresión del antígeno Ki67 como índice de proliferación celular y comparar los hallazgos con los observados en el CVB en matrices de tejidos (TMA).

\section{MATERIAL Y MÉTODO}

Casos. Se seleccionaron 96 casos de vesículas biliares con colecistitis crónica, sin evidencias de reagudización inflamatoria, signos de regeneración epitelial, displasia, ni tumor, del Servicio de Anatomía Patológica de Clínica Alemana de Santiago y 102 CVB del Hospital de Temuco, Chile. En todos los casos el diagnóstico se realizó en la pieza de colecistectomía. El nivel de infiltración tumoral fue establecido mediante el estudio seriado de la totalidad de la vesícula biliar (mapeo) de acuerdo a protocolos establecidos desde hace más de una década y previamente publicados ${ }^{23,24}$.

Confección de matrices de tejidos (TMA). Se seleccionaron 3 áreas representativas de cada una de las vesículas biliares no tumorales y de los CVB. Para la confección de las matrices, se utilizó el "Semiautomatic Tissue MicroArrayer Pathology Devices, Inc." con agujas de $2 \mathrm{~mm}$. Los 3 cilindros de tejidos fueron extraídos del bloque donante previa marcación de las láminas teñidas con $\mathrm{HE}$ y puestos en el bloque receptor a una distancia de $3 \mathrm{~mm}$ de separación. Las inclusiones fueron puestas en estufa a $55^{\circ} \mathrm{C}$ por 30 min para obtener la adherencia entre las muestras y el bloque receptor.

Técnica de inmunohistoquímica. Se realizó la técnica estándar para tejidos fijados en formalina e incluidos en parafina. Los cortes histológicos de los TMA de 4 micras de espesor fueron desparafinados e hidratados en concentraciones decrecientes de alcohol. La recuperación de antígenos se realizó mediante exposición a microondas en buffer citrato $\mathrm{pH}$ 6,0 y lavados en PBS pH 7,4. Se utilizó anticuerpo anti Ki67 ("monoclonal antibody clone MIB-1 Dako ${ }^{\mathrm{TM}}$ ") en dilución 1/ 100. El anticuerpo primario fue incubado a tempera- 
tura ambiente por 60 min y luego incubado con el complejo "Super Picture Polymer Detection $\mathrm{Kit}^{\mathrm{TM}}$ Zymed" en equipo Dakoautostainer $^{\mathrm{TM}}$.

Formas de medición de la positividad. Se consideró como expresión positiva a la tinción nuclear. La intensidad de la tinción positiva se evaluó en escala visual desde claramente perceptible + o débil, ++ moderada, hasta +H intensa. Los casos dudosos fueron considerados negativos. Como control interno positivo se empleó la tinción de células epiteliales, estromales o linfoides positivas. El conteo se realizó en las áreas de mayor positividad. La medición de la positividad de los casos fue realizada por dos observadores y fue clasificada en base al porcentaje de positividad de las células epiteliales no tumorales como tumorales.

Estadística. Se realizó mediante test de chi cuadrado y exacto de Fisher para las tablas de contingencia.

\section{RESULTADOS}

Características generales del grupo estudiado. La distribución por sexo y edad de los 198 casos en estudio (96 casos de MNT y 102 CVB), se resumen en la Tabla 1. En el grupo de MNT 77\% (74 casos) comespondieron a mujeres, con un promedio de edad de 44,6 años ( $\mathrm{DE} \pm 15,8$ años) y los casos restantes a hombres, con una edad promedio de 53,4 (DE $\pm 16,2$ años). El grupo de CVB constituido por $82 \%$ de mujeres con un promedio de edad de 61,7 años ( $\mathrm{DE} \pm 14,4$ años) y los casos restantes por hombres con una edad promedio de 69,8 (DE $\pm 13,2$ años). La diferencia de los promedios de edad entre hombres y mujeres fue significativa en ambos grupos ( $p \varangle 0,001$ ).

Resultados estudio inmunohistoquímico. El porcentaje de positividad del Ki67 en MNT y en el CVB se resume en la Tabla 2. En la MNT el índice de marcación fue de 18,8\% con límites entre 0\% y

Tabla 1. D istribución por género y promedios de edad de pacientes con vesículas biliares no tumorales y portadores de cáncer vesicular

\begin{tabular}{|lcccccc|}
\hline & \multicolumn{2}{c}{ Mucosa no tumoral } & \multicolumn{3}{c|}{ Cáncer vesícula biliar } \\
& Femenino & Masculino & Total & Femenino & M asculino & Total \\
\hline N & 74 & 22 & 96 & 84 & 18 & 102 \\
Edad promedio & 44,6 & 53,4 & 46,5 & 61,7 & 69,8 & 62,5 \\
DE & 15,8 & 16,2 & 16,2 & 14,4 & 13,2 & 13,4 \\
& & & & & & $\mathrm{p}<0,001$ \\
\hline
\end{tabular}

DE =Desviación estándar. Edad =años

Tabla 2. D istribución porcentual de la positividad de Ki67 en vesículas biliares con mucosa no tumoral y cáncer de la vesícula biliar

\begin{tabular}{|c|c|c|c|c|c|c|}
\hline \multirow[b]{2}{*}{ Positividad \% } & \multicolumn{2}{|c|}{ MNT } & \multicolumn{2}{|c|}{ Cáncer } & \multirow[b]{2}{*}{ \% no cáncer } & \multirow[b]{2}{*}{ \% cáncer } \\
\hline & $\mathbf{n}$ & $\%$ & $\mathbf{n}$ & $\%$ & & \\
\hline 0 to 10 & 52 & 54,7 & 9 & 5,9 & 85 & 15 \\
\hline 10 to 20 & 12 & 12,6 & 30 & 19,6 & 29 & 71 \\
\hline 20 to 30 & 8 & 8,4 & 19 & 12,4 & 30 & 70 \\
\hline 30 to 40 & 6 & 6,3 & 17 & 11,1 & 26 & 74 \\
\hline 40 to 50 & 3 & 3,2 & 17 & 11,1 & 15 & 85 \\
\hline 50 to 60 & 6 & 6,3 & 9 & 5,9 & 40 & 60 \\
\hline 60 to 70 & 5 & 5,3 & 9 & 5,9 & 36 & 64 \\
\hline 70 to 80 & 2 & 2,1 & 13 & 8,5 & 13 & 87 \\
\hline 80 to 90 & 1 & 1,1 & 12 & 7,8 & 8 & 92 \\
\hline 90 to 100 & 0 & 0,0 & 18 & 11,8 & 0 & 100 \\
\hline
\end{tabular}

MNT $=$ Mucosa no tumoral 
89\% (DE $\pm 24,7 \%)$ y los CVB tuvieron una positividad significativamente mayor de $46 \%$ con límites entre 2,6\% y 98\% (DE $\pm 29,4)$ ( $p$ $<0,001)$.

La localización de la tinción positiva en la MNT se observó sólo en las porciones superficiales, en la mayoría de los casos (Figuras $1 \mathrm{~A}$ y B), siendo esta área la de mayor proliferación celular en la mucosa vesicular en comparación a las zonas profundas, en donde se observó expresión de Ki67 en forma ocasional y aislada. La constitución de estructuras glandulares más complejas con grupos glandulares o acinos, se observó sólo en relación a focos de metaplasia gástrica o intestinal. En estos casos la positividad se observó en las zonas más profundas de la mucosa, lo cual fue interpretado como un cambio en la localización de la zona proliferativa de la MNT (Figuras $1 \mathrm{C}$ y D).

En la MNT 54,7\% expresó el antígeno Ki67 entre $0 \%$ y $10 \%$ de las células epiteliales de la mucosa, en cambio, sólo 5,9\% de los CVB estuvieron en ese rango (Tabla 2). En porcentajes mayores a $10 \%$ los casos de MNT disminuyeron significativamente con un predominio de CVB.

Al clasificar la positividad en grupos de $10 \%$ e incluir tanto a los casos de MNT como a los CVB, se observa que en el grupo de $0 \%$ a $10 \%$ de positividad, $85 \%$ del total de casos corresponden a mucosa no tumoral (52 MNT y 9 CVB). Este porcentaje se invierte en el grupo inmediatamente superior (positividad entre $10 \%$ y $20 \%$ ), en donde $71 \%$ de los casos correspondieron a CVB (30 CVB y $12 \mathrm{MNT}$ ). A partir de $10 \%$ de positividad la expresión de Ki67 fue más frecuentemente observada en los CVB que en lesiones no tumorales (Figura 2).

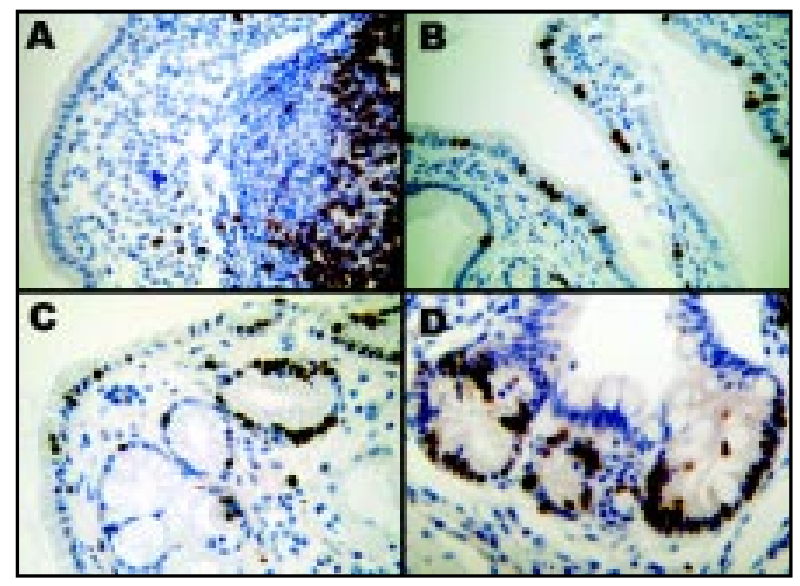

Figura 1. Expresión de Ki67 en la mucosa de la vesícula biliar no tumoral. Se observa en las imágenes superiores, positividad nuclear intensa en un acúmulo linfoide en la mucosa y negatividad en el epitelio (A). Positividad focal en el epitelio vesicular normal (B). Positividad en las estructuras glandulares en áreas de metaplasia de tipo gástrico (C) e intestinal (D).

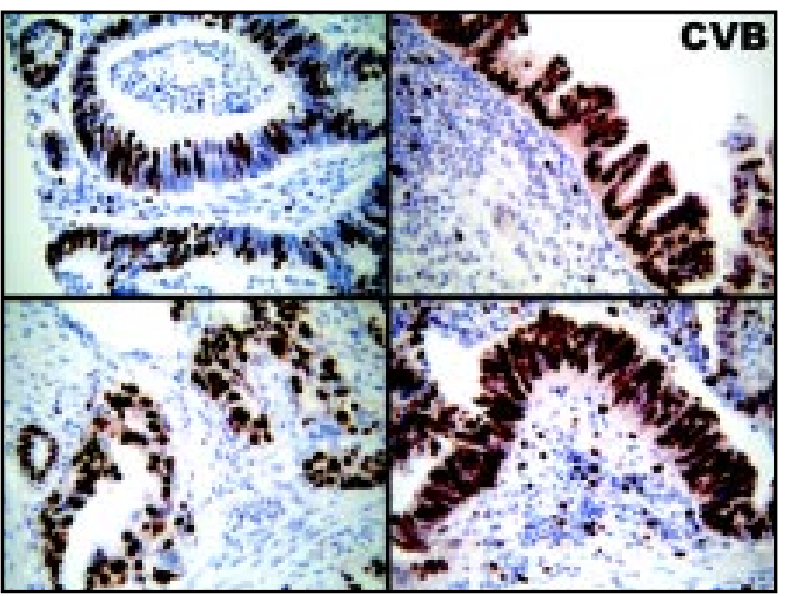

Figura 2. Expresión de Ki67 en distintos tipos de adenocarcinoma de la vesícula biliar. Intensa positividad en el epitelio tumoral, con porcentajes de entre $40 \%$ hasta casi $100 \%$ de las células epiteliales tumorales.

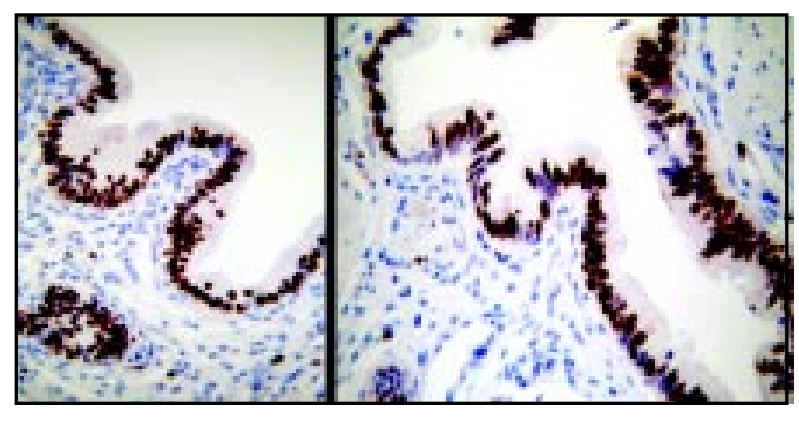

Figura 3. Expresión de Ki67 en mucosa vesicular no tumoral con intensa positividad en la mayoría de las células del epitelio vesicular normal sobre $80 \%$. Nótese la ausencia de inflamación o signos de regeneración, lo cual traduciría un estado «iperproliferativo» con la mayoría de las células epiteliales en fases activas del ciclo celular. 
Por su parte los CVB, tuvieron porcentajes de positividad significativamente mayores que los de MNT, con una distribución más homogénea en todos los grupos porcentuales en comparación a la MNT. Sólo 5,9\% de los CVB tuvieron una positividad menor de $10 \%$ en las células tumorales; en cambio, $75 \%$ de los CVB tuvieron una positividad superior a 20\% (Tabla 2).

Es llamativo que casi 15\% de las MNT mostraron índices de expresión de Ki67 mayores a 50\%, y $25 \%$ mayor de $20 \%$, es decir, porcentajes francamente anormales para una mucosa del tubo digestivo (Figura 3). En éstos se observa a la MNT en estado de hiperproliferación del epitelio, con una alta cantidad de células epiteliales en fases activas del ciclo celular.

$\mathrm{Si}$ se considera como un porcentaje aceptable para un tejido lábil normal de alrededor de $20 \%$ de expresión de Ki67, 33\% de las vesículas biliares crónicamente inflamadas de nuestros pacientes estarían por sobre esta cifra.

\section{DisCUSIÓN}

La proliferación celular, medida a través de la expresión del antígeno Ki67 ha sido extensamente estudiada y validada tanto en tejidos normales como tumorales. La comparación con otras técnicas capaces de determinar el porcentaje de células en fases activas del ciclo celular ha sido extensa- mente reportada ${ }^{25-27}$. Sin embargo, la interpretación de los índices de fijación de Ki67 debe realizarse en comparación con tejidos normales, en consideración a la marcada variación que tienen los distintos histiones. Así, el tejido cere$\mathrm{bral}^{28}$, mama ${ }^{29}$ y otros tienen porcentajes de Ki67 que rara vez sobrepasan el 5\%. Otros tejidos como estómago ${ }^{30}$, colon ${ }^{31}$, esófago ${ }^{32} \mathrm{y} \mathrm{piel}^{27}$ presentan índices de proliferación algo mayores.

Los escasos estudios realizados en la mucosa vesicular no tumoral con Ki67, muestran porcentajes de positividad de entre $1 \%$ y $10 \%$ en series que fluctúan entre 6 y 11 casos, lo cual limita significativamente la validez de las observaciones ${ }^{16,33-35}$ (Tabla 3). Sin embargo, en todos los trabajos este porcentaje es significativamente menor que el encontrado en nuestros casos, lo cual pudiese significar que las células epiteliales de la mucosa vesicular crónicamente inflamada en nuestros pacientes tendrían mayor actividad proliferativa, lo que pudiese guardar relación con la alta frecuencia de litiasis, inflamación crónica y cáncer vesicular en nuestra población.

El 85\% de las MNT mostraron índices de Ki67 menores de $20 \%$ y el $70 \%$ de los cánceres mayores de $10 \%$, lo cual demuestra una clara separación entre lesiones tumorales y no tumorales en base a los índices de proliferación medidos a través de la expresión de Ki67. Los CVB presentan índices de proliferación significativamente mayores que las MNT (46,1\% v/s 18,7\%). Sin embargo, a lo menos

Tabla 3. Índices de positividad de Ki67 reportados en la literatura en M N T, adenomas y CVB

\begin{tabular}{|lcccc|}
\hline Diagnóstico & \% positividad & DE (\%) & N casos & Ref \\
\hline CVB & 36,6 & 5,6 & 8 & $(39)$ \\
& 44,5 & 5,4 & 3 & $(34)$ \\
& 25,8 & 16,4 & 41 & $(40)$ \\
& 68,7 & 10,2 & 13 & $(16)$ \\
& 38,4 & 11,6 & 4 CIS & $(16)$ \\
& 50,0 & & 55 & $(41)$ \\
Adenomas & 26,8 & 5,8 & 12 & $(42)$ \\
& 10,3 & & 78 & $(43)$ \\
MNT & 7,6 & 3,0 & 8 & $(42)$ \\
& 1,0 & 1,0 & 9 & $(12)$ \\
& 1,2 & 1,5 & 6 & $(34)$ \\
& 6,1 & 12,0 & 6 & $(35)$ \\
\hline
\end{tabular}


$15 \%$ de las VB no tumorales presentan índices de proliferación celular dentro del rango propio de los CVB. La existencia de este grupo hace suponer la presencia de pacientes con un alto grado de proliferación celular, alteración que pudiese ser la base a la aparición de lesiones de carácter cualitativo y cuantitativo de la diferenciación celular como son las metaplasias y displasias de la vesícula biliar respectivamente.

No existe aún información respecto de factores y vías a través de las cuales se produciría el

\section{REFERENCIAS}

1. SERRA I. [Has gallbladder cancer mortality decrease in Chile?]. Rev Méd Chile 2001; 129: 1079-84.

2. ANdia KM, GederLini GA, FerReccio RC. [Gallbladder cancer: trend and risk distribution in Chile]. Rev Méd Chile 2006; 134: 565-74.

3. Lazcano-Ponce EC, Miquel JF, Munoz N, Herrero R, FerRecio C, Wistuba II, et al. Epidemiology and molecular pathology of gallbladder cancer. CA Cancer J Clin 2001; 51: 349-64.

4. PANDEy M. Risk factors for gallbladder cancer: a reappraisal. Eur J Cancer Prev 2003; 12: 15-24.

5. Andia ME, Hsing AW, Andreotti G, Ferreccio C. Geographic variation of gallbladder cancer mortality and risk factors in Chile: a population-based ecologic study. Int J Cancer 2008; 123: 1411-6.

6. Wistuba, II, GaZDar AF. Gallbladder cancer: lessons from a rare tumour. Nat Rev Cancer 2004; 4: 695-706.

7. Misra S, Chaturvedi A, Misra NC, Sharma ID. Carcinoma of the gallbladder. Lancet Oncol 2003; 4: 167-76.

8. Kodama $\mathrm{K}$, Nakadaira $\mathrm{H}$, Endoh $\mathrm{K}$, Yamamoto M. Geographic clustering patterns in mortality from biliary tract cancer in Japan. Jpn J Cancer Res 1998; 89: 6-11.

9. Kayahara M, Nagakawa T. Recent trends of gallbladder cancer in Japan: an analysis of 4,770 patients. Cancer 2007; 110: 572-80.

10. Roa I, de Aretxabala X, Araya JC, Roa J. Preneoplastic lesions in gallbladder cancer. J Surg Oncol 2006; 93: 615-23.

11. Yamagiwa $H$, Tomiyama $H$. Intestinal metaplasia-dysplasia-carcinoma sequence of the gallbladder. Acta Pathol Jpn 1986; 36: 989-97.

12. Vakkala M, Laurita JJ, Saarnio J, Koivukangas V, Syrjala H, Karttunen T et al. Cellular turnover and expression of hypoxic-inducible factor in acute acalculous and calculous cholecystitis. Crit Care 2007; 11: R116. estímulo proliferativo ${ }^{36,37}$. La irritación crónica, el estrés oxidativo producto de la inflamación y la presencia de una bilis mutagénica ${ }^{38}$ pudiesen determinar estados de mayor actividad proliferativa con un mayor riesgo de transformación neoplásica.

Se requieren estudios genético-moleculares de la mucosa vesicular normal, crónicamente inflamada, metaplasias y displasias para avanzar en la comprensión de la patogenia de esta importante neoplasia en nuestro país.

13. Lamote J, WiLems G. DNA synthesis, cell proliferation index in normal and abnormal gallbladder epithelium. Microsc Res Tech 1997; 38: 609-15.

14. Putz P, Wiшems G. Cell proliferation in the human gallbladder epithelium: effect of distension. Gut 1979; 20: 246-8.

15. Isozaki $\mathrm{H}$, Okajima $\mathrm{K}$, Hara $\mathrm{H}$, Sako $\mathrm{S}$, Mabuchi $\mathrm{H}$. Proliferating cell nuclear antigen expression in the gallbladder with pancreaticobiliary maljunction. J Surg Oncol 1997; 65: 46-9.

16. LEE CS. Differences in cell proliferation and prognostic significance of proliferating cell nuclear antigen and Ki-67 antigen immunoreactivity in situ and invasive carcinomas of the extrahepatic biliary tract. Cancer 1996; 78: 1881-7.

17. Roa I, Araya J, Shiraishi T, Yatani R, Viliaseca M, Wistuba I ET AL. Proliferating cell nuclear antigen in gallbladder carcinoma. Histopathology 1993; 23: 179-83.

18. Yanagisana N, Mikami T, Koike M, Okayasu I. Enhanced cell kinetics, p53 accumulation and high p21WAF1 expression in chronic cholecystitis: comparison with background mucosa of gallbladder carcinomas. Histopathology 2000; 36: 54-61.

19. Sai JK, Suyama M, Nobukawa B, Kubokawa Y, Yokomizo $\mathrm{K}$, Sato N. Precancerous mucosal changes in the gallbladder of patients with occult pancreatobiliary reflux. Gastrointest Endosc 2005; 61: 264-8.

20. Colozza M, Azambuja E, Cardoso F, Sotiriou C, LarsiMONT D, PICCART MJ. Proliferative markers as prognostic and predictive tools in early breast cancer: where are we now? Ann Oncol 2005; 16: 1723-39.

21. Stuart-Harris R, Caldas C, Pinder SE, Pharoah P. Proliferation markers and survival in early breast cancer: a systematic review and meta-analysis of 85 studies in 32,825 patients. Breast 2008; 17: 323-34.

22. Rindi G, D’Adda T, Froio E, Feluegara G, Bordi C. Prognostic factors in gastrointestinal endocrine tumors. Endocr Pathol 2007; 18: 145-9. 
23. Roa I, Araya JC, Wistuba I, de AretXabala X. [Gallbladder cancer: anatomic and anatomo-pathologic considerations]. Rev Méd Chile 1990; 118: 572-9.

24. Roa I, Araya JC, Vilaseca M, Roa J, de Aretxabala X, IвACACHe G. Gallbladder cancer in a high risk area: morphological patterns. Hepatogastroenterology 1999; 46: 1540-6.

25. Goodson WH, $3^{\text {RD }}$, Moore DH, $2^{\text {ND }}$, LJung BM, CHew K, FLORENDO C, MAYALL B ET AL. The functional relationship between in vivo bromodeoxyuridine labeling index and Ki-67 proliferation index in human breast cancer. Breast Cancer Res Treat 1998; 49: 155-64.

26. Bozlu M, Orhan D, Baltaci S, Yaman O, Elhan AH, TulunaY O ET AL. The prognostic value of proliferating cell nuclear antigen, Ki-67 and nucleolar organizer region in transitional cell carcinoma of the bladder. Int Urol Nephrol 2002; 33: 59-66.

27. Heenen M, Thiriar S, Noel JC, Galand P. Ki-67 immunostaining of normal human epidermis: comparison with $3 \mathrm{H}$-thymidine labelling and PCNA immunostaining. Dermatology 1998; 197: 123-6.

28. Lu G, WaI SM, Poon WS, Yew DT. Ki67 and doublecortin positive cells in the human prefrontal cortices of normal aging and vascular dementia. Microsc Res Tech 2005; 68: 255-7.

29. Urruticoechea A, Smth IE, Dowsett M. Proliferation marker Ki-67 in early breast cancer. J Clin Oncol 2005; 23: 7212-20.

30. Sougioultzis S, Foukas PG, Tzivras M, Kourtessas D, Gorgoulis VG, Davaris $P$ ET aL. Alterations in the proliferating compartment of gastric mucosa during Helicobacter pylori infection: the putative role of epithelial cells expressing p27(kip1). Mod Pathol 2003; 16: 1076-85.

31. Holt PR, Moss SF, Kapetanakis AM, Petrotos A, Wang $\mathrm{S}$. Is Ki-67 a better proliferative marker in the colon than proliferating cell nuclear antigen? Cancer Epidemiol Biomarkers Prev 1997; 6: 131-5.

32. Jaskiewicz K, De Groot KM. p53 gene mutants expression, cellular proliferation and differentiation in oesophageal carcinoma and non-cancerous epithelium. Anticancer Res 1994; 14: 137-40.

33. Noda Y, Fujta N, Kobayashi G, Ito K, Horaguchi J, TAKASAWA $O$ ET AL. Histological study of gallbladder and bile duct epithelia in patients with anomalous arrangement of the pancreaticobiliary ductal system: companison between those with and without a dilated common bile duct. J Gastroenterol 2007; 42: 211-8.

34. Tanno S, Obara T, Fuji T, Mizukami Y, Yanagana N, IzAWA T ET AL. Epithelial hyperplasia of the gallbladder in children pancreaticobiliary ductal union. Hepatogastroenterology 1999; 46: 3068-73.

35. Tanno S, Obara T, Fujil T, Mizukami $Y$, Shudo $R$, Nishino N ET AL. Proliferative potential and K-ras mutation in epithelial hyperplasia of the gallbladder in patients with anomalous pancreaticobiliary ductal union. Cancer 1998; 83: 267-75.

36. Ai Z, Lu W, Q IN X. Arsenic trioxide induces gallbladder carcinoma cell apoptosis via downregulation of Bcl-2. Biochem Biophys Res Commun 2006; 348: 1075-81.

37. Tsuchida A, Itoi T, Aoki T, Koyanagi Y. Carcinogenetic process in gallbladder mucosa with pancreaticobiliary maljunction (Review). Oncol Rep 2003; 10: 1693-9.

38. Mano H, Roa I, Araya JC, Ohta T, Yoshida K, Araki K ET AL. Comparison of mutagenic activity of bile between Chilean and Japanese female patients having cholelithiasis. Mutat Res 1996; 371: 73-7.

39. Takei K, Watanabe H, Itoi T, Saito T. p53 and Ki-67 immunoreactivity and nuclear morphometry of 'carcinoma-in-adenoma' and adenoma of the gallbladder. Pathol Int 1996; 46: 426-35.

40. Hidalgo Grau LA. Gallbladder carcinoma: the role of p53 protein overexpression and Ki67 antigen expression as prognostic factor. HPJ 2004; 6: 174-80.

41. Li SH, L CF, Sung MT, ENg HL, Hsiung CY, Huang WW ET AL. Skp2 is an independent prognosticator of gallbladder carcinoma among p27(Kip1)-interacting cell cycle regulators: an immunohistochemical study of 62 cases by tissue microarray. Mod Pathol 2007; 20: 497-507.

42. Jin YM, Yim H, Shim C. Cyclin E expression in benign and malignant epithelial neoplasms of the gallbladder. Yonsei Med J 1997; 38: 301-6.

43. Nagata S, Ajioka Y, Nishikura K, Watanabe G, Inoue T, YAMAGUCHI K ET AL. Co-expression of gastric and biliary phenotype in pyloric-gland type adenoma of the gallbladder: immunohistochemical analysis of mucin profile and CD10. Oncol Rep 2007; 17: 721-9. 